\title{
A Case Study on the Spatial Panel Data Model : Factors of China Marine Economy Development
}

\author{
Hong Mianmian ${ }^{1, a^{*}}$ \\ ${ }^{1}$ School of Economics \& Commerce, South China University of Technology, China \\ a miaomian522@163.com
}

Keywords: Marine economy, Factors, Spatial panel data model.

\begin{abstract}
Entering the 21st century, China's marine economy has been experiencing rapid development, and the spatial structure of coastal city's marine economy has also changed significantly. This paper, introducing the term "geospatial", chooses a selection of index, with consideration of spatial effects, regarding the factors that affect the regional development of China's marine economy. Based on the panel data of 11 coastal cities and provinces in China between 2000 and 2014, ordinary panel data model and spatial panel data model are constructed to analyze quantitatively the impact of these factors on the regional development of marine economy under different spatial scales. The analysis indicates that spatial Durbin model with the application of spatial effect can make more accurate estimation. The result suggests that capital investment, labor forces, natural resource and foreign direct investment have positive effects on the marine economic growth in China.
\end{abstract}

\section{我国海洋经济区域发展的影响因素分析}

---基于空间面板数据模型的实证研究

\author{
洪绵绵 $1, a^{*}$ \\ 1华南理工大学经济与贸易学院, 广州, 中国 \\ amiaomian522@163.com
}

关键词：海洋经济; 影响因素; 空间面板数据模型

中文摘要. 进入 21 世纪后, 我国海洋经济飞速发展, 沿海省市海洋经济的空间格局也发生了 显著变化。本文将 “地理空间” 引入区域海洋经济的分析中, 在考虑空间效应的基础上, 根 据目前我国海洋经济区域发展现状选取相应的影响因素指标, 采用2000-2014年我国沿海11个 省市的面板数据，构建普通面板数据模型和空间面板数据模型对这些影响因素在不同时空尺 度上对海洋经济区域发展的作用强度进行定量研究。实证显示引入空间效应的空间杜宾模型 能更准确地估计结果, 结果表示资本投入、劳动力水平、自然资源禀赋和外商直接投资等因 素对我国海洋经济增长有正向的推动作用。

\section{1. 引言}

进入21世纪后, 海洋经济作为海陆双向发展的综合性经济, 已经成为各国经济持续增长 的巨大动力, 也将是全球经济发展最具潜力的方向。随着我国区域经济一体化进程的不断加 快, 作为陆域经济系统不可分割的一部分, 海洋经济区域发展会受周边地区的辐射影响, 沿 海各省市之间的海洋经济增长和海洋产业发展等方面的空间集聚及溢出效应的作用越来越显 
著。因此，在考虑空间效应的基础上研究海洋经济发展的影响因素问题，有利于更深层次地 把握我国沿海11个省市海洋经济发展的互动关系, 对海洋经济可持续发展和中国经济长期协 调发展具有十分重要的理论意义和实践意义。

关于我国海洋经济区域发展的影响因素分析方面，张向前、欧阳钦芬（2001） ${ }^{[1]}$ 认为影 响海洋经济区域发展的主要因素有自然资源、劳动力资源、资本、技术、结构变化及制度安 排等, 除上述因素外, 马雯月（2008） [2]还提出了金融要素与管理要素也是导致海洋经济区 域性差异的重要因素。董夏（2013） [3]则定性地分析了自然基础、陆域经济基础、海洋空间 集聚、政策投资等因素。在其基础上，常玉苗（2011） ${ }^{[4]}$ 构建普通面板数据模型进行实证分 析, 得出海洋产业规模、港口数量及政策因素对海洋经济发展影响显著。贾宁（2011） ${ }^{[5]}$ 通 过构建普通面板数据模型探索海洋经济区域差异性出现的原因, 认为科技水平、劳动力、人 力资本、外商直接投资对海洋经济区域增长有促进作用。贾明瑶（2012）[6]则在深入分析江 苏省海洋经济差异的内在动力机制后得出劳动力水平、地区资本积累、外商直接投资、交通 通达性等因素对海洋经济区域发展起重要作用。纵观已有文献, 中国海洋区域经济的研究多 忽略了空间因素。但在分析区域经济问题时, 应当把空间效应这一关键因素纳入经济模型中 具体研究。为了解决这种区域间的相互影响, Anselin (1988) ${ }^{[7]}$ 、Anselin与Rey (1991) ${ }^{[8]}$ 提出 了空间计量模型。于是一些国外学者将空间计量分析方法引入到地区经济增长收敛研究中来

(Rey和Montouri，1999; Rey和Dev，2004）[9][10]。随着空间经济计量手段的成熟, 国内学者 也将空间效应引入到区域经济差异的趋同分析中，例如林光平、龙志和等（2005） ${ }^{[11]}$ 采用空 间经济计量方法来分析 1978-2002年我国 28 个省市实际人均GDP的 $\beta$-收敛情况; 张学良 (2009) ${ }^{[12]}$ 根据长三角132个县市区的统计数据, 运用空间经济计量的方法对区域经济增长收 敛性问题进行实证研究。

通过以上文献的整理, 对于海洋经济区域发展的影响因素分析, 已有研究不仅分析依据 和重点各有侧重，而且基本是采用定性描述分析，基于面板数据的区域增长分析鲜有涉及。 进一步来看, 中国海洋区域经济的研究基本上是在忽略空间效应的基础上展开的, 因此空间 计量经济方法的引入能为我国海洋经济相关问题的探究带来全新的视角。

\section{2. 变量选取与模型构建}

在2000-2014年间，推动我国海洋经济区域发展的动力机制是什么以及这些因素的影响程 度是多少等问题尚未明确。进一步可发现, 各个区域并非独立的个体, 其经济发展会受相邻 区域的辐射作用，那么我国海洋经济活动的空间效应又是如何作用的。因此，本文引入空间 效应, 通过建立普通面板数据模型和空间计量经济模型, 对我国海洋经济区域差异的影响因 素进行回归分析，比较引入空间效应前后的结果，以期可以得出更贴近现实的结论。

\section{1 变量选取与数据说明}

依据文献的整理分析可得, 影响我国沿海省市海洋经济发展的因素有自然资源禀赋、陆 域经济发展基础、产业空间集聚、政府政策与投资等等。本文根据我国沿海省市海洋经济发 展的实际情况以及模型的需要, 兼顾数据的易获取性和可量化性, 选取表 1 中的指标对海洋经 济区域发展产生的影响因素进行探究。 
表1 衡量我国海洋经济区域发展影响因素的变量选取

\begin{tabular}{|c|c|}
\hline 变量 & 变量说明 \\
\hline 人均海洋经济产 (M0) & 模型的因变量, 可测度我国沿海各省市海洋经济的区域发展。 \\
\hline 资本投入（MK） & $\begin{array}{l}\text { 各沿海省市全社会固定资产投资/GDP, 资本投入通过乘数效应积极推 } \\
\text { 动各地区的技术、劳动力、资本等要素发展。 }\end{array}$ \\
\hline 劳动力水平 $(M L)$ & $\begin{array}{l}\text { 选取各沿海省市涉海就业人员这一指标来衡量沿海各地区海洋经济 } \\
\text { 发展的劳动力水平 }\end{array}$ \\
\hline 科技进步指数（MT） & $\begin{array}{l}\text { 采用平均赋权法, 归一化处理各沿海省市的科研机构数量、科研从业 } \\
\text { 人员、科技机构课题数量三个指标数据后乘以权重, 再相加 }\end{array}$ \\
\hline 自然资源禀赋（MR） & $\begin{array}{l}\text { 选取海水养殖面积来表示。这一指标能较好地反映各沿海省市的自然 } \\
\text { 资源状况, 与海洋经济产值密切相关, 单位为公顷。 }\end{array}$ \\
\hline 外商直接投资（MF） & $\begin{array}{l}\text { 外商直接投资可衡量一个地区区位条件的优越性, 对区域经济差异的 } \\
\text { 影响巨大，数据选用外商投资企业年底注册登记的投资总额 }\end{array}$ \\
\hline
\end{tabular}

注: 作者整理所得。

本文研究所需要的数据均来自《中国海洋统计年鉴》、《中国海洋经济统计公报》、《中 国统计年鉴》以及相关沿海省市海洋统计年鉴。研究对象是 11 个沿海省市: 天津市、河北省、 辽宁省、上海市、江苏省、浙江省、福建省、山东省、广东省、广西省、海南省, 研究时间 段是2000-2014年。最终的面板数据包含了15年间 11 个截面单位的 165 个样本。由于选取相关 指标时各个变量的单位和口径不一样, 因此本文对所有指标数据进行归一化处理, 得到处于 0-1之间的数值, 减小误差。

\section{2 模型设定}

本文所涉及的模型如下:

(1) 普通面板数据模型的设定

普通面板数据模型不考虑空间效应，主要是与空间计量模型进行比较分析。具体模型如 下:

$$
M O_{i t}=\beta_{0}+\beta_{1} * M K_{i t}+\beta_{2} * M L_{i t}+\beta_{3} * M T_{i t}+\beta_{4} * M R_{i t}+\beta_{5} * M F_{i t}+\varepsilon_{i t}
$$

其中, $\beta$ 是回归系数, $\varepsilon$ it为随机误差项。

(2) 空间面板数据模型的设定

Ansel et al.(2008)研究发现如果当样本存在空间自相关性时，传统的普通面板数据模型所 估计出来的结果存在偏差, 并不能准确地度量各个变量之间的关系, 而应该引入空间效应, 建立空间模型。空间模型分为空间滞后模型（SAR）、空间误差模型（SEM）和空间杜宾模 型（SDM）。空间杜宾模型（SDM）包含因变量和自变量空间自相关性，是空间误差模型和 空间滞后模型的扩展形式, 可以通过对空间滞后模型和空间误差模型增加相应的约束条件设 立。该模型的基本构造如下:

$$
Y_{t}=\rho W Y_{t}+X_{t} \beta+\kappa W X_{t} \beta+u_{t}
$$

其中, $\mathrm{W}$ 为空间权重矩阵。如果对空间杜宾模型（SDM）增加约束条件 $\kappa=0$ ，则空间杜 宾模型（SDM）则转化成空间滞后模型（SAR），如果对空间杜宾模型（SDM）增加约束条 件 $\kappa=-\rho$ ，则空间杜宾模型（SDM）则转化成空间误差模型（SEM）。

普通面板模型和空间面板模型选择的标准取决于Robust LM-lag和Robust LM-err检验, 具 体公式如下:

$$
\begin{gathered}
\text { Robust }-L M-1 \text { ag }=\frac{\left[e^{T}\left(I_{T} \otimes W\right) Y / \sigma^{2}-e^{T}\left(I_{T} \otimes W\right) e / \sigma^{2}\right]^{2}}{J-T T_{w}} \\
\text { Robust }-L M-\text { error }=\frac{\left[e^{T}\left(I_{T} \otimes W\right) Y / \sigma^{2}-T T_{w} / J \times e^{T}\left(I_{T} \otimes W\right) Y / \sigma^{2}\right]^{2}}{T T_{w}\left[1-T T_{w} / J\right]}
\end{gathered}
$$


其中, $J=\frac{1}{\sigma^{2}}\left[\left(\left(I_{T} \otimes W\right) X \beta\right)^{T}\left(I_{N T}-X\left(X^{T} X\right)^{-1} X^{T}\right)\left(I_{T} \otimes W\right) X \beta+T T_{w} \sigma^{2}\right], T_{w}=\operatorname{tr}\left(W W+W^{T} W\right)$ ， $\otimes$ 克罗内克积, $e$ 和 $\beta$ 为普通面板模型估计出来的残差序列和回归系数, $\sigma$ 为普通面板模型 的回归标准误差, $I_{T}$ 为 $T \times T$ 维的单位矩阵, $T$ 为期限数, $\operatorname{tr}()$ 为该矩阵的迹。Robust LM-lag 和 Robust LM-err 检验均服从卡方分布。

本文的建模思路如下: 先对普通面板数据模型进行估计, 再进行Robust LM-lag和Robust LM-err检验。若Robust LM-lag和Robust LM-err都不显著, 则选择普通面板数据模型; 若Robust LM-lag和Robust LM-err有一个显著, 则选择空间杜宾模型。之后对杜宾模型若做Wald spatial lag和Wald spatial error检验以确认模型的正确选择, 即分别对两个约束条件 $\kappa=0$ 和 $\kappa=-\rho$ 进 行验证, 若 $\kappa=0$ 无法被拒绝, 则选择空间滞后模型, 若 $\kappa=-\rho$ 无法被拒绝, 则选用空间误 差模型。最后进行Hausman检验决定模型是固定效应还是随机效应。

本文选取的空间权重矩阵是基于地理距离函数关系设定的，具体形式如下：

$$
W_{i j}= \begin{cases}0 & (i=j) \\ \frac{1}{d_{i j}} & (i \neq j)\end{cases}
$$

其中, $d_{i j}$ 是 $i$ 和 $j$ 省省会城市的球面距离。

\section{3. 我国海洋经济区域发展影响因素的实证研究}

借助Matlab7.0软件, 对2000-2014年我国沿海省市海洋经济区域发展的影响因素进行普通 面板数据模型的OLS回归分析，结果如表2。

由表 2 可知，回归方程的拟合优度为 0.1691 , 拟合程度较低。从回归结果的系数来看，在 $5 \%$ 的显著性水平下, 资本投入 (MK) 和劳动力水平 (ML) 这两个变量均通过检验, 且系数 为正。自然资源禀赋 (MR) 和外商直接投资 (MF) 的系数为正, 但未通过 $\mathrm{P}$ 值检验, 这可能 是模型的拟合程度较低, 估计结果出现偏差, 这可待下一步检验。然而, 科技进步指数 (MT) 与人均海洋经济产值 (MO) 成反比, 这可能和相关技术投入的滞后性有关, 有待进一步研究。

从表2的实证结果可以发现：（1）我国沿海11个省市海洋经济估计出来的LR检验结果显 示, 在 $1 \%$ 的显著性水平之下, 我们的研究样本存在个体特殊效应。（2）在LM检验中, Robust LM Lag和Robust LM Err均在 $5 \%$ 的显著性水平下拒绝原假设, 且显著性程度高, 由此可见普 通面板数据模型估计的结果不能准确地度量不同因素对我国海洋经济区域差异的影响程度, 因此我们需要引入空间效应，构建空间计量模型来进行实证分析。

表2 2000-2014年我国海洋经济影响因素分析的实证结果

\begin{tabular}{|c|c|c|c|}
\hline \multicolumn{2}{|c|}{ 普通面板数据模型 } & \multicolumn{2}{|c|}{ 空间杜宾模型 } \\
\hline 变量及检验统计量 & 回归系数 & 变量及检验统计量 & 回归系数 \\
\hline MR & 0.068942 & MR & $0.132025^{* *}$ \\
\hline MT & -0.062806 & MT & -0.065741 \\
\hline MK & $0.158160^{\text {**** }}$ & MK & $0.084626^{* *}$ \\
\hline $\mathrm{ML}$ & $2.961451^{* * * *}$ & $\mathrm{ML}$ & $2.350951^{* * *}$ \\
\hline MF & 0.015153 & MF & $0.173619^{* *}$ \\
\hline & & W*MR & $37.586561^{*}$ \\
\hline & & $\mathrm{W} * \mathrm{MT}$ & -19.266952 \\
\hline & & $\mathrm{W} * \mathrm{MK}$ & $46.781888^{* * * 4}$ \\
\hline & & $\mathrm{W} * \mathrm{ML}$ & 171. $457037^{*}$ \\
\hline & & $\mathrm{W} * \mathrm{MF}$ & $-28.023880^{*}$ \\
\hline & & W*y & -1.000000 \\
\hline$\overline{\mathrm{R}^{2}}$ & 0.1691 & $\overline{R^{2}}$ & 0.9432 \\
\hline LR-test & $391.7723^{\text {**** }}$ & Hausman test & $65.0448^{* * * *}$ \\
\hline Robust LM Lag & $10.3096^{* * *}$ & Wald lag & $17.9773^{\text {weter }}$ \\
\hline Robust LM Err & $7.7751^{* * * *}$ & Wald err & $19.7053^{* * * *}$ \\
\hline
\end{tabular}


注: *表示在 $10 \%$ 的显著性水平下显著，**表示在5\%的显著性水平下显著，***表示在1\%的显著性水

平下显著。

根据上述的分析结果并结合空间杜宾模型（2-2），借助Matlab7.0软件，对2000-2014年 我国沿海 11 个省市海洋经济的空间活动进行估计, 其实证结果如表2所示。Wald检验结果显 示, Wald lag和 Wald err在 $1 \%$ 的显著性水平下均拒绝原假设, 这表明本文选择空间杜宾模型 是正确的。Hausman检验结果显示，本文的空间杜宾模型在 $1 \%$ 的显著性水平下拒绝 $\mathrm{h}=0$ 的原 假设，应该选择固定效应系数。

在考虑了空间效应后，即将空间杜宾模型的估计结果和普通面板数据模型的估计结果进 行对比分析, 探究空间效应对我国沿海省市区域发展的影响, 可以得出如下结论: 引入空间 效应对普通面板数据模型起到了很好的优化效果, 且各变量的系数得到了改善。空间杜宾模 型中的 $\mathrm{R}^{2}$ 为 0.9432 , 相对于 OLS模型的估计结果, 拟合优度非常高。对比表 2 , 引入空间效应 后各变量系数都出现了变化，且与预期基本一致。自然资源禀赋（MR）、资本投入（MK）、 劳动力水平 (ML) 和外商直接投资 (MF) 对我国沿海11个省市人均海洋经济产值的影响系 数均为正, 虽然科技进步指数（MT）的系数为负，但没有通过 $10 \%$ 的显著性水平检验，其结 果有偏差。进一步可发现, 自然资源禀赋（MR）和外商直接投资（MF）在OLS回归下，其 结果没有通过 $10 \%$ 的显著性水平检验, 而引入空间效应后, 其结果变得显著, 除了科技进步 指数（MT）外，其余 4 个变量的统计结果均通过了 $5 \%$ 的显著性水平检验。这说明了忽略空间 效应, 其估计结果会出现偏差, 使得不同因素对我国沿海省市海洋经济的影响程度产生误差。

根据表2的实证结果来具体探究我国沿海省市海洋经济区域发展的影响因素, 我们可以发 现:

（1）劳动力水平 (ML) 的影响系数为 2.350951 , 是各项变量中系数最大的, 且通过 $1 \%$ 的显著性水平检验。这表明劳动力水平在我国沿海省市海洋经济区域发展的影响因素中占主 导地位。目前, 我国仍处于海洋经济欠发达阶段, 海洋产业主要以资源密集型和劳动密集型 为主, 在一定程度上我国沿海省市海洋经济的发展是劳动拉动型的增长模式。因此, 各沿海 省市不能忽视海洋专业劳动力的培养和投资, 提高海洋产业劳动力水平是我国各沿海省市保 持海洋经济持续发展的关键。

(2) 外商直接投资 (MF) 的影响系数是 0.173619 , 且该系数通过了 $5 \%$ 的显著性水平检验。 这说明外商直接投资对我国海洋经济的增长有正向的推动作用，其投资的产业倾斜会直接影 响行业的分工格局和区域经济的发展差异化。随着海洋经济的快速发展, 外部资本大量进入 沿海地区。其投资一般集中在高附加值的海洋高新技术新兴产业上, 这也有利于我国沿海省 市海洋经济产业结构的转型升级。

（3）海水养殖面积能较好地反映各沿海省市的自然资源状况, 与海洋经济产值密切相关。 在 $5 \%$ 的显著性水平下, 用海水养殖面积表示的自然资源禀赋（MR）的影响系数为 0.132025 , 与人均海洋经济产值成正相关。这表明自然资源禀赋是我国海洋经济区域差异的重要因素。 目前我国海洋经济的发展仍处在以利用自然资源为主的阶段, 所以不同地区的海洋资源丰富 程度会直接导致各省市海洋产业体系的构建，进而影响海洋经济区域发展水平。

(4) 从各省市资本积累方面来看, 实证结果表明其对海洋经济区域发展的影响相对较小。 资本投入 (MK) 的影响系数是 0.084626 , 且通过 $5 \%$ 的显著性水平检验。经济基础较好的地 区可以更有效地配置资源, 吸引更多先进要素, 优先得到政府政策支持, 全社会固定投资也 不断增加, 形成良性循环。但是资本投入的作用更多体现在陆域经济的发展上, 通过陆域经 济的联动作用影响海洋产业的发展, 从而其对海洋经济增长的影响相对较小。这也进一步表 明我国海洋经济的发展不是资本密集型。

(5) 在实证结果中, 科技进步指数 (MT) 的影响系数为 -0.065741 , 与人均海洋经济产值 成负相关，对海洋经济发展有反向作用。这可能和科技技术的滞后性有关，也可能是由于选 取各沿海省市的科研机构数量、科研从业人员、科技机构课题数量作为参考指标不够科学。 且该系数无论是在普通面板数据模型中还是空间经济计量模型都没有通过显著性水平检验, 因此科学进步指数这一指标有待进一步研究分析。 


\section{4. 结论}

本文将“地理空间”引入区域海洋经济的分析中，在考虑空间效应的基础上,采用2000-2014 年我国沿海 11 个省市的面板数据, 构建普通面板数据模型和空间面板数据模型对这些影响因 素在不同时空尺度上对海洋经济区域发展的作用强度进行定量研究, 结果发现引入空间效应 对普通面板数据模型起到了很好的优化效果, 且各变量的系数得到了改善, 即考虑空间效应 的空间杜宾模型优于未考虑空间效应的普通面板数据模型。根据空间杜宾模型的估计结果， 劳动力水平、外商直接投资、自然资源禀赋和资本投入的影响系数均为正，且通过了 $5 \%$ 的显 著性水平检验, 这表明其对我国海洋经济增长有正向的推动作用。而科技进步指数的影响系 数为负, 这可能与科学技术的滞后性有关, 也可能是本文在评价科技影响时选取的指标不够 科学。但这一系数未通过显著性水平检验, 因此这一指标有待进一步研究。

\section{References}

[1] Zhang Xiangqian, Ouyang Qinfen. The analysis of marine economy and regional economic development. Asia-Pacific Economic Review. 2002(2) : 44-47

[2] Ma wenyue. The analysis of factors that put ocean economy forward. China Water Transport.Vol.8 No.01 January 2008.

[3] Dong Sha. The analysis on the space-time diversity of China's marine economic regional system[D].Liaoning Normal University,2013

[4] CHANG Yu miao. A case study on China's coastal province's panel data: factors of oceanic economic development.Resources\&Industries,2011,13(5).

[5] Jia Ning. The testing and the influence factors analyzing of regional disparity of marine economy[D].Ocean University of China,2011.

[6] Jia Mingyao. Empirical analysis on the spatial difference and dynamic mechanism of marine economy development in Jiangsu[D].Nanjing University,2012.

[7] Anselin,L. Spatial econometrics: Methods and models. Kluwer Academic Publishers, Dordrecht,1988.

[8] Anselin,L., Rey, S. Properties of tests for spatial dependence in linear regression models[J]. Geographical Analysis, 1991,23:112-131

[9] Rey, S. J., Montouri, B. D. (1999) US regional income convergence: aspatial econometric perspective. Regional Studies, 33: 143-156

[10]Rey, S., B. Dev. Sigma-convergence in the presence of spatial effects[R].Urban/Regional 0404008, EconWPA, 2004

[11]Guangping Lin, Zhihe Long, Mei Wu. A spatial analysis of regional economic convergence in China:1978-2002.China Economic Quarterly.Vol.4,Supp.Oct.,2005.

[12]Zhang Xue-liang. A Spatial Econometrics Analysis of Regional Economic Convergence in China: Based on the Empirical Study of 132 Counties, Cities or Regions in Yangtze River Delta from 1993 to 2006.Journal of Finance and Economics. Vol.35 No.7 Jul.2009. 